\title{
Implementing Quantile Selection Models in Stata
}

\author{
Mariel Siravegna \\ Ercio Munoz \\ Georgetown University \\ The Graduate Center, CUNY
}

July 30, 2020 
- A simple sample selection model can be written as the latent model

$$
Y^{*}=X^{\prime} \beta+\mu
$$

but $Y^{*}$ is only observed if $\mathrm{S}=1$

$$
S=\mathbf{1}\left(Z^{\prime} \gamma+v \geq 0\right)
$$

- Since the seminal work of Heckman (1979), much progress has been made in methods that extend the original model or relax some of its assumptions

- And recently Arellano and Bonhomme (2017) proposed a copula-based method to correct for sample selection in quantile regression 
Two Recent Applications 
- In this paper the authors use the CPS between 1976-2013 to see how the gender wage gap vary across the wage distribution

- They assess how selective participation of individuals in the labor market affects the gender gap 
Comparison of Female and Male Wage CDF

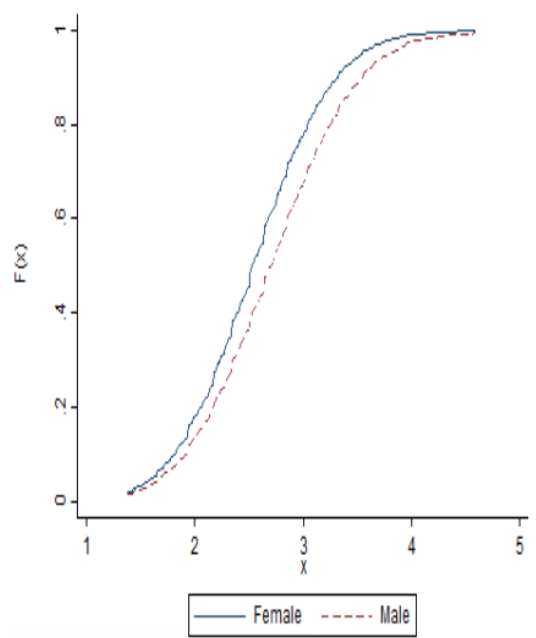

(Without correction)

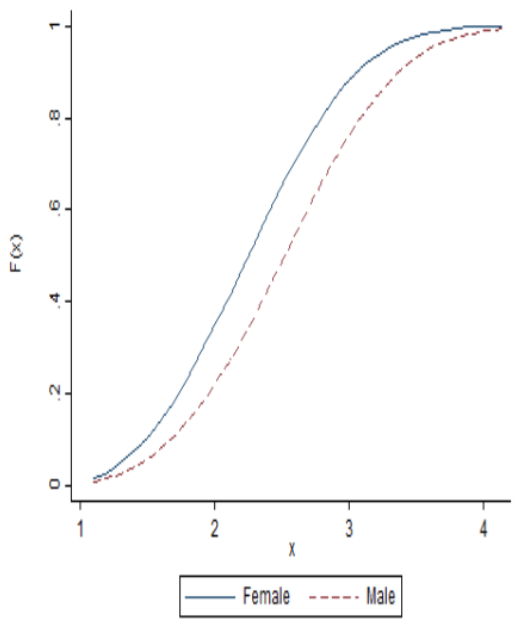

(Correcting for Selection) 
- Survey earnings response is not random

- In this paper the authors match the survey earnings responses to administrative records to see how response vary across the earnings distribution

- They find that non-response rate follows an $U$ shape across earnings and this produces an underestimation of inequality, which can be corrected using this copula-based approach 


\section{Bollinger et al. (JPE 2019)}

TABLE 7

Performance of Selection Correction Methods

FOr NonRESPONSE IN THE ASEC ON INEQUALITY

\begin{tabular}{lcccr}
\hline \hline & \multicolumn{3}{c}{ INEQUALITY MEASURES } \\
\cline { 2 - 5 } SAMPLE & Gini & $90-10$ & $90-50$ & $50-10$ \\
\hline ASEC & .461 & 10.099 & 2.607 & 3.870 \\
ASEC, only respondents with IPW & .464 & 10.227 & 2.641 & 3.869 \\
ASEC, only respondents with copula & .482 & 10.521 & 2.676 & 3.929 \\
ASEC for respondents, DER for & & & & \\
$\quad$ nonrespondents (benchmark) & .477 & 11.038 & 2.683 & 4.112 \\
\hline
\end{tabular}




\section{Estimation}


Given an i.i.d sample $\left(Y_{i}, Z_{i}, S_{i}\right), i=1, \ldots, N$ where $Z_{i}=\left(X_{i}, W_{i}\right)$ and assuming that quantile functions are linear:

$$
q(\tau, x)=x^{\prime} \beta_{\tau}, \quad \text { for all } \tau \in(0,1) \text { and } x \in X
$$

the algorithm is as follows:

1. Estimation of the propensity score $p(z)$

2. Estimation of the dependence parameter or degree of selection $\rho$ using this moment restriction:

$$
\mathbb{E}\left[I\left(Y \leq X^{\prime} \hat{\beta}_{\tau}\right)-G(\tau, p(z) ; \rho) \mid S=1, Z=z\right]=0
$$




\section{Second Step}

Taken to the sample by choosing a $\rho$ that minimizes the following objective function:

$$
\hat{\rho}=\operatorname{argmin}_{\rho}\left\|\sum_{i=1}^{N} \sum_{l=1}^{L} S_{i} \varphi_{\tau_{l}}\left(z_{i}\right)\left[I\left\{Y_{i} \leq X_{i}^{\prime} \tilde{\beta}_{\tau_{l}}(\rho)\right\}-G\left(\tau_{l}, p\left(z_{i}^{\prime}\right) ; \rho\right)\right]\right\|
$$

where $\|$.$\| is the Euclidean norm, \tau_{1}<\tau_{2}<\cdots<\tau_{L}$ is a finite grid on $(0,1)$, and the instrument functions are defined as $\varphi_{\tau_{l}}\left(z_{i}\right), G\left(\tau_{l}, p\left(z_{i}^{\prime}\right) ; \rho\right)$ is the conditional copula indexed by a parameter $\rho$, and:

$$
\tilde{\beta}_{\tau}(\rho)=\operatorname{argmin}_{\beta} \sum_{i=1}^{N} S_{i}\left[G_{\tau_{i}}\left(Y_{i}-X_{i}^{\prime} \beta\right)^{+}+\left(1-G_{\tau, i}\left(Y_{i}-X_{i}^{\prime} \beta\right)^{-}\right]\right.
$$

where $a^{+}=\max \{a, 0\}, a^{-}=\max \{-a, 0\}$, and $G_{\tau, i}=G(\tau, p(z) ; \rho)$. 
3. Given the estimated $\hat{\rho}, \hat{\beta}_{\tau}$ can be estimated by minimizing a rotated check function of the form:

$$
\hat{\beta_{\tau}}=\operatorname{argmin}_{\beta} \sum_{i=1}^{N} S_{i}\left[\hat{G_{\tau, i}}\left(Y_{i}-X_{i}^{\prime} \beta\right)^{+}+\left(1-\hat{G_{\tau, i}}\right)\left(Y_{i}-X_{i}^{\prime} \beta\right)^{-}\right]
$$

where $\hat{\beta}_{\tau}$ will be a consistent estimator of the $\tau$-th quantile regression coefficient.

Note that this step is unnecessary if the researcher is interested on the quantiles included in the finite grid of step 2 . 


\section{Implementing the method in Stata}




\section{Syntax}

qregsel depvar [indepvars] [if $][$ in $], \operatorname{select}\left(\left[\operatorname{depvar}_{S}=\right]\right.$ varlist $\left._{S}\right)$

quantile(\#) grid_min(grid_minvalue) grid_max (grid_maxvalue)

grid_length(grid_lengthvalue) [ copula(copula) noconstant plot ] 


\section{Empirical Example}




\section{Wages of women used in Heckman command}

- global wage_eqn wage educ age

- global seleqn married children educ age

- qregsel \$wage_eqn, select(\$seleqn) quantile(.1 .5 .9) copula(gaussian) ///

$>$ grid_min(-.9) grid_max(.9) grid_length(.05) plot

Quantile selection model

Number of obs

1343

\begin{tabular}{r|rrr} 
& q10 & q50 & q90 \\
\hline education & 1.083723 & 1.017025 & .8888879 \\
age & .204362 & .2028979 & .2272004 \\
-cons & -8.148793 & .5828089 & 8.914994
\end{tabular}




\section{Grid for minimization}

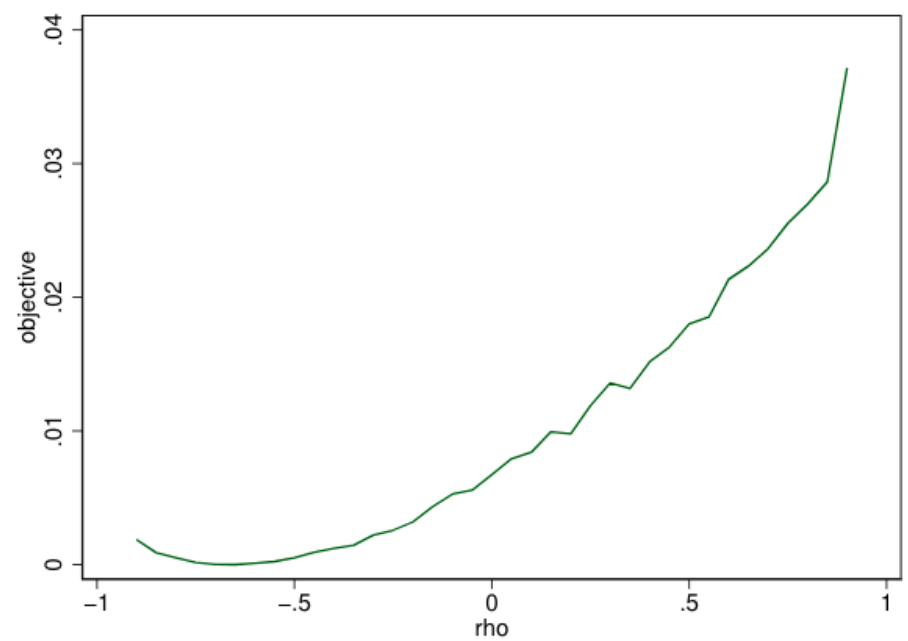

Figure 2: Grid for minimization 


\section{Counterfactual distribution: Corrected versus uncorrected quantiles}

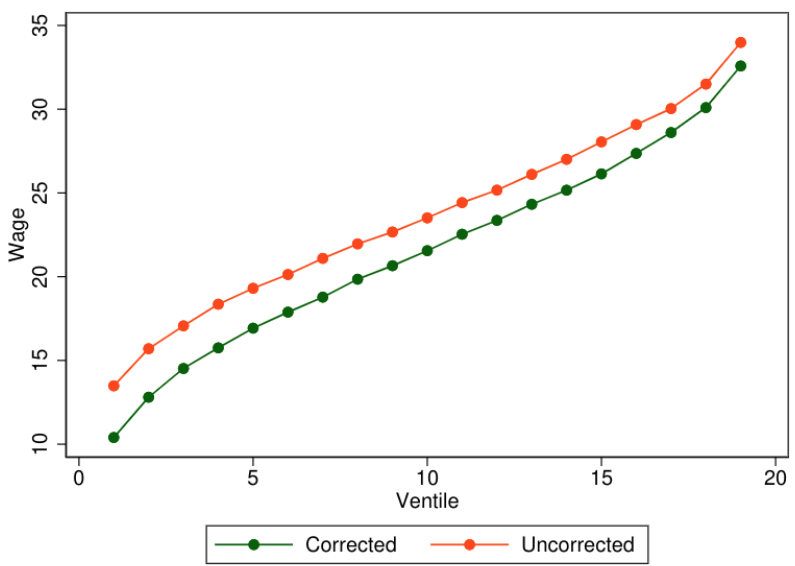

Figure 3: Corrected versus uncorrected quantiles 


\section{Conclusions}


- We have introduced a new Stata command that implements a copula-based method to correct for sample selection in quantile regressions proposed in Arellano and Bonhomme (2017)

- This command may be useful for Stata users doing empirical work, as we have illustrated with the case of two recently published papers

- The code is for now only available in our github repo

- Questions, comments, and suggestions are welcome 
- Arellano, M., and S. Bonhomme (2017), "Quantile Selection Models with an Application to Understanding Changes in Wage Inequality." Econometrica 85(1)

- Bollinger, C., B. Hirsch, C. Hokayem, and J. Ziliak (2019), "Trouble in the Tails? What We Know about Earnings Nonresponse Thirty Years after Lillard, Smith, and Welch." Journal of Political Economy 127(5).

- Maasoumi, E., and L. Wang (2019), "The Gender Gap between Earnings Distributions." Journal of Political Economy 127(5).

- Munoz, E., and M. Siravegna (2020), "Implementing Quantile Selection Models in Stata." 